\title{
Distribution of acid mucus in the bronchial mucous glands
}

\author{
N. KOLLERSTROM, P. W. LORD, AND W. F. WHIMSTER
}

From the MRC Environmental Hazards Unit, St. Bartholomew's Hospital Medical College, Charterhouse Square, London EC1M $6 B Q$

Kollerstrom, N., Lord, P. W., and Whimster, W. F. (1977). Thorax, 32, 160-162. Distribution of acid mucus in the bronchial mucous glands. The percentage area of the bronchial glands taking up the Alcian blue ( $\mathrm{pH} \mathrm{2.6)} \mathrm{stain} \mathrm{for} \mathrm{acid} \mathrm{mucin} \mathrm{was} \mathrm{obtained} \mathrm{from} \mathrm{sections} \mathrm{from} \mathrm{the}$ trachea and from each generation down the inferior lingular axial bronchial pathway in five non-smokers and five cigarette smokers. The mean percentage value for the smokers $(59.5 \%)$ was significantly less than for the non-smokers $(74 \cdot 4 \%)$. The percentage increased between successive generations from the trachea by an average of 6.5 . This gradient did not differ significantly between the smoking and non-smoking groups. It was not possible to attribute these differences to any specific mechanism because many interacting variables were involved which could not be measured. Nevertheless the percentage mucin retention or storage provides an introduction to consideration of these variables and their response to cigarette smoke.

Examination of sections from human trachea and bronchi during investigations into bronchial mucous gland hypertrophy (Whimster et al., in preparation) suggested that the proportion of acid mucus in these glands was greater towards the more distal airways. We therefore measured the amount of intraglandular acid mucus in each generation of branching down the inferior lingular axial pathway in a group of 10 patients.

\section{Material and methods}

The left lung with the trachea was obtained at postmortem examination from each of 10 patients, five non-smokers and five cigarette smokers (Table 1). None of the patients had died of illnesses involving the respiratory system, except case e who had oat-cell carcinoma of the right lung.

The entire airway from the trachea (counted as G0) to the fifth generation (G5) of the inferior lingular pathway was dissected out. For the trachea and main bronchus, longitudinal sections of tissue, some $12 \mathrm{~mm}$ long including two or three cross-sections of cartilage rings, were taken. Two such sections were taken from the middle part of the main bronchus (G1) and three from each of the upper, middle, and lower trachea. Transverse sections of tissue were taken from the middle of each generation from G2 (lobar bronchus) to G5.
Table 1 Age, sex, and smoking histories of the 10 cases

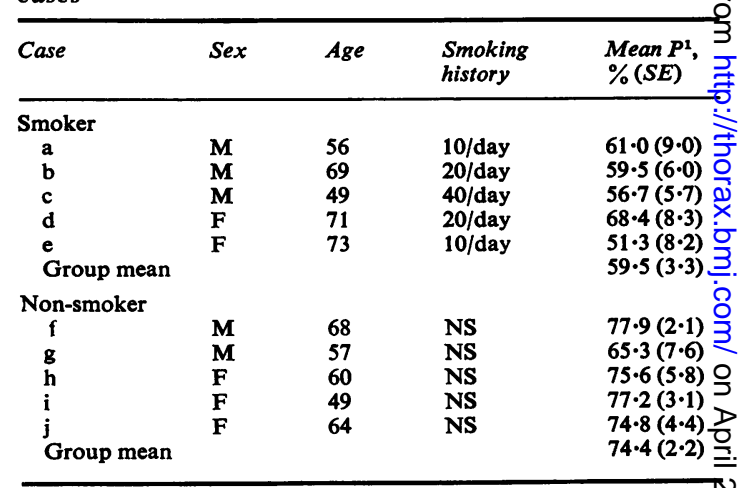

The mean value of the proportion of acid mucus in the mucous glands $(P)$ is tabulated.

NS $=$ non-smoker.

After routine processing and embedding in wað one section $5 \mu \mathrm{m}$ thick was taken from eack block and stained with Alcian blue at $\mathrm{pH} 2.6$ for 30 minutes, followed by Cole's haematoxylin fom 10 minutes, and with $1 \%$ watery eosin for 6 mini utes (AB 2.6/HE). By this method the cells of the subepithelial mucous glands could be identified microscopically and within these the blue staining intraglandular acid mucus could easily be seef and distinguished from mucin on the luminat surface or in ducts. 
The sections were examined using a light microscope with $\times 25$ and $\times 40$ objectives and $\times 10$ eyepieces. Areas of the subepithelial mucous glands which were Alcian blue negative $(\mathrm{g})$ and areas which were Alcian blue positive $(\mathrm{m})$ were estimated separately by counting the number of points superimposed on them by the intersections of an eyepiece graticule with a $0.5 \mathrm{~mm}$ square lattice. The percentage of acid mucus in the total gland was estimated by the ratio $P=100 \mathrm{~m} /(\mathrm{m}+\mathrm{g})$.

This proportion was obtained at the three tracheal sites and at each generation in each case, except for case $h$ at $G 3$ and for case $d$ at G3 and G5. Estimates at these sites could not be made because the tissue was too autolysed. For G4 and G5 whole sections were counted, while in the more proximal airways, where there was more gland tissue, randomly sampled gland clusters were counted. Counts were generally between 100 and 1000 for each component, although sometimes in peripheral generations one component was too sparse and a count of less than 100 had to be accepted.

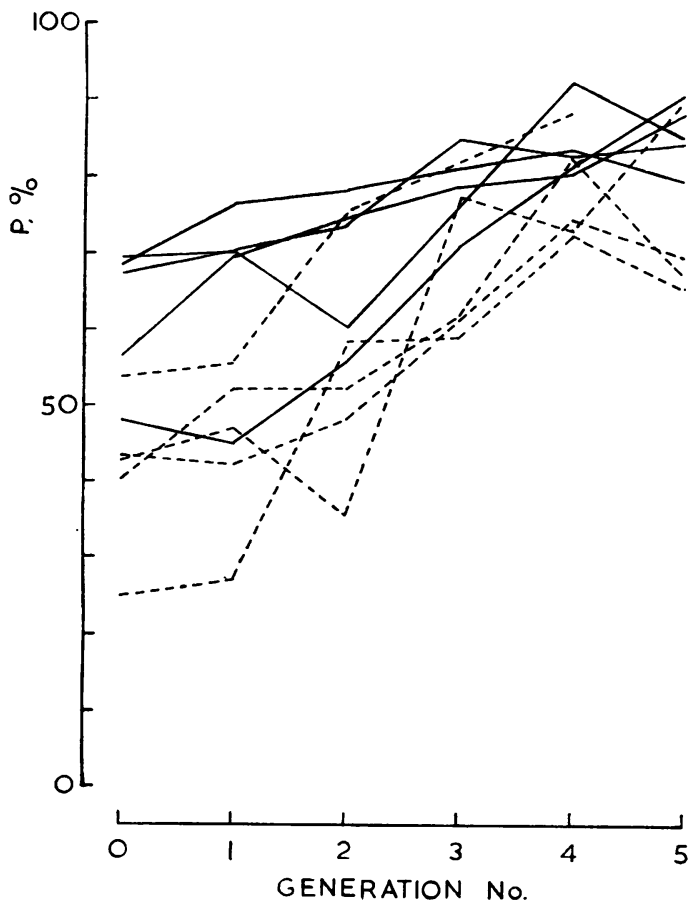

(a)
Results

No significant differences were seen between the three tracheal sites so these counts were pooled to obtain a proportion P for the whole of G0. In Fig. 1a the proportion of mucus in the glands, $P$, is plotted at each generation in each case.

An analysis of variance was performed on the values of $P$ (Table 2) taking as factors smoking habit, sex, and regression on generation. This showed a significant difference $(P<0.001)$ between the non-smoking and smoking groups. The nonsmokers had proportionately more intraglandular acid mucus, the mean value being $74.4 \%$, against the smokers' mean of $59.5 \%$. The ratio did not vary significantly between males and females.

The interaction term in the analysis between the regression on generation number and the smoking groups was not significant, which indicated that separate regressions on generation number within each of the two categories (smokers and non-smokers) were not significantly different; the regression coefficient for the smokers was

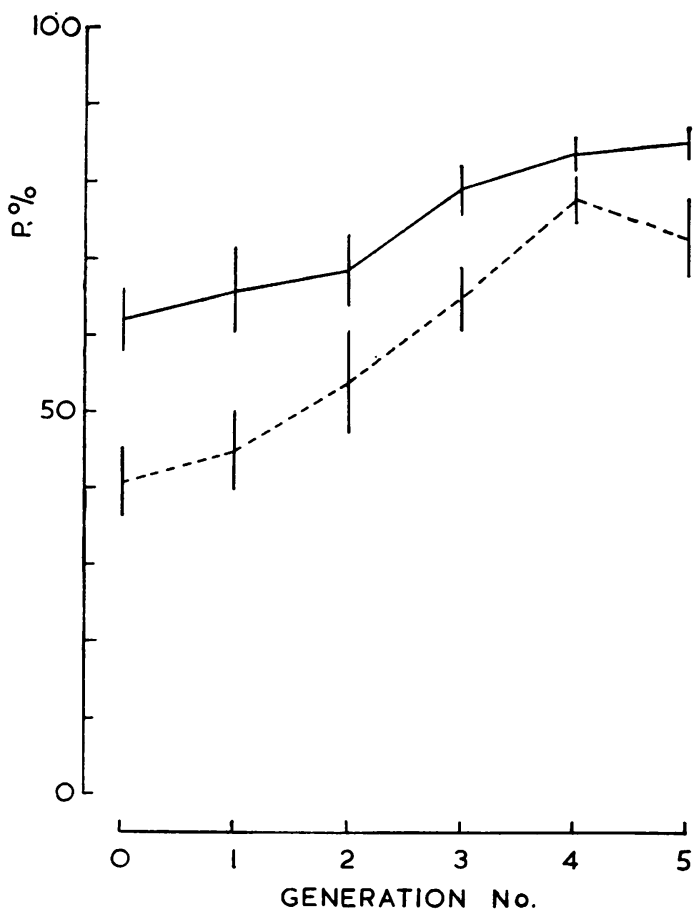

(b)

Fig. 1 (a) Values of $P=100 \mathrm{~m} /(\mathrm{m}+\mathrm{g})$, percentage acid mucus in mucous glands, in each case: non-smokers $\longrightarrow$; smokers -----. (b) Mean values and standard errors of $P$ for non-smokers $\stackrel{\longrightarrow}{\longrightarrow}$ and smokers $(----)$. 
Table 2 Analysis of variance for $P$

\begin{tabular}{|c|c|c|c|}
\hline Source of variation & $\begin{array}{l}\text { Degrees of } \\
\text { freedom }\end{array}$ & $\begin{array}{l}\text { Mean sum } \\
\text { of squares }\end{array}$ & $\begin{array}{l}\text { Variance ratio } \\
\text { (over residual) }\end{array}$ \\
\hline Smoking group & 1 & $0 \cdot 3318$ & $35 \cdot 54$ \\
\hline Sex & 1 & 0.0114 & $1 \cdot 22$ \\
\hline \multirow{2}{*}{\multicolumn{4}{|c|}{$\begin{array}{l}\text { Smoking } \times \text { sex } \\
\text { Regression on }\end{array}$}} \\
\hline & & & \\
\hline generation number & 1 & $0 \cdot 7351$ & $78 \cdot 73$ \\
\hline \multicolumn{4}{|l|}{ Deviations from } \\
\hline regression & 4 & 0.0118 & $1 \cdot 26$ \\
\hline Smoking $\times$ regression & 1 & $0 \cdot 0289$ & $3 \cdot 10$ \\
\hline \multicolumn{4}{|l|}{ Smoking $\times$ deviations } \\
\hline from regression & 4 & 0.0033 & $0 \cdot 37$ \\
\hline Residual & 43 & 0.0093 & \\
\hline
\end{tabular}

slightly higher than for non-smokers. Taking both groups together, a significant $(P<0.001)$ linear regression on generation number was obtained, $P$ increasing towards the distal part of the airway by $6.5( \pm 0.7) \%$ per generation (regression \pm standard error). The absence of significant variation due to deviations from the regression on generation number showed that a linear term only on generation number was adequate to describe the trend.

\section{Discussion}

This is the first time that acid mucin has been studied in relation to the whole mass of cells (excluding ducts) of which bronchial glands are composed. Our initial observation had suggested that there were relatively more cells and less mucin in the more proximal airways, and this study formally confirms this impression. The results show that the proportion of gland which takes up the stain for acid mucin increases by an average of $6.5 \%$ with each successive generation down the lingular pathway, whether the patient is a smoker or not.

There was no suggestion that there were more connective tissue or inflammatory or other nonsecreting cells in the more proximal glands, so the proximal secretory cells as a whole must have contained relatively less acid mucin and relatively more cytoplasm.

Within a single mucous cell the rate of change of mucus volume, $\frac{d v}{d t}$, within it will be the difference between the rate of production, $\frac{d p}{d t}$, of mucus and the rate of excretion, $\frac{\mathrm{de}}{\mathrm{dt}}$, of mucus from the cell, both of which may vary with time. The volume of mucus within the cell at any particular time, $t$, after the cell was formed will be the integral of the rate of change of volume over time plus the initial amount of mucus present, $V_{1}$, (if any):

$$
V=\int_{0}^{t}\left(\frac{d p}{d t}-\frac{d e}{d t}\right) d t+V_{I}
$$

The individual mucous cells within a gland will vary in their rates of production and excretion $\vec{\sigma}_{\bar{\sigma}}$ and in their initial volumes. At any given time the volume of intraglandular mucin will be the sumis of the individual contributions, each given by an $\vec{\circ}$ equation such as that shown above. If these cells: function independently of each other one would $\vec{\omega}$ expect the total volume of mucus in a gland to? show only minor fluctuations about a mean value $x$ with time. Similarly, the total volume of a gland w will be given by the sum of all the individual celli volumes, which may themselves be varying, and $\vec{\sigma}$ one would expect this volume to be stable too. WeO have related the former to the total volume at one? particular time, in this case the time of death. From these data alone we cannot say in what way the distributions of the ratio (P) of mucus volume to the total gland which we have observed were $\overrightarrow{0}$ reached or how they were changing with time. $\checkmark$ Neither can we relate the proportion we have measured to average production and excretion? rates in the different airways.

Decreasing concentrations of particles ando gases in the inspired air in successive generationso

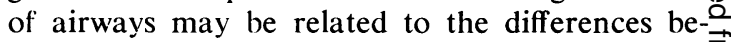
tween the upper and the more distal large airways.ô In this connection it is therefore interesting that in smokers the trend in $P$ with generation number is still observed but lies below that for nonsmokers at all generations (Fig. 1b). These lower values of $P$ are associated with the more hypertrophied glands of smokers (Ryder et al., 1971).

We are grateful to Professor P. J. Lawther, Director, MRC Environmental Hazards Unit, for advice and encouragement, and also to Janet Edwards for help in the preparation of this paper.? We should also like to thank Dr. R. Salm, con $\frac{7}{0}$ sultant histopathologist, Royal Cornwall Hospital, and Dr. J. L. Pead, consultant histopathologist, N Basildon Hospital. for supplying postmortem material.

\section{Reference}

Ryder, R. C.. Dunnill, M. S., and Anderson, J. A.त्ष (1971). A quantitative study of bronchial mucous? gland volume, emphysema and smoking in at necropsy population. Journal of Pathology. 104.0 $59-71$.

Requests for reprints to: P. W. Lord, MRC Environ $\stackrel{\mathbb{D}}{2}$ mental Hazards Unit, St. Bartholcmew's Hospita最 Medical College, Charterhcuse Square, Londono ECIM 6BQ. 